\title{
A Kinematic Human-Walking Model for the Normal-Gait-Speed Estimation Using Tri-Axial Acceleration Signals at Waist Location
}

\author{
Jwu-Sheng Hu*, Member, IEEE, Kuan-Chun Sun, and Chi-Yuan Cheng
}

\begin{abstract}
This study aims at estimating the human walking speed using wearable accelerometers by proposing a novel virtual inverted pendulum model. This model not only keeps the important characteristics of both the biped rolling-foot and the inverted pendulum model, but also makes the speed estimation feasible using human body acceleration. Rather than using statistical methods, the proposed kinematic walking model enables calibration of the parameters during walking using only one tri-axial accelerometer on the waist that collects the user's body acceleration. In addition, this model also includes the effect of rotation of the waist within a walking cycle, which improves the estimation accuracy. Experimental results for a group of humans show a $0.58 \%$ absolute error mean and $0.72 \%$ error deviation, which is far better than the results of other known studies with accelerometers mounted on the upper body.
\end{abstract}

Index Terms-Gait, human mechatronics, inertia sensor, kinematic walking model, walking speed, wearable sensor.

\section{INTRODUCTION}

$\mathbf{T}$ HE use of accelerometers worn on the body to quantify human movement is increasingly popular in recent years. Task-specific studies that use single or multiple bodymounted accelerometers were reported to quantitatively measure the functional performance of gait cycles [1], [2]. While acceleration data are useful for examining basic temporal-spatial gait parameters for clinical applications, they also provide personal activity information that benefits technological development in health care and pervasive computing [3]. Among the various gait parameters estimated from body acceleration, walking speed can also be used as an odometer for localization for humans (e.g., combined with a wireless sensor network). It performs better than step counts using a pedometer [4]. The authors in [5] collected some researches and carried out a comparison of five methods of accelerometer-based estimation of the step length in straight-line human walking.

There are two different kinds of methods of estimating walking speed: signal based and model based. The signal-based approaches use either statistical regression with offline parameters calibration or training via an artificial neural network (ANN).

Manuscript received August 2, 2012; revised December 4, 2012 and February 3, 2013; accepted March 4, 2013. Date of publication March 15, 2013; date of current version July 13, 2013. Asterisk indicates corresponding author.

${ }^{*} \mathrm{~J}$.-S. Hu is currently a Professor in the Department of Electrical and Control Engineering, National Chiao Tung University, Hsinchu 300, Taiwan, ROC (e-mail: jshu@cn.nctu.edu.tw).

K.-C. Sun and C.-Y. Cheng are with the Department of Electrical and Control Engineering, National Chiao Tung University, Taiwan, ROC (e-mail: gcs.ece98g@nctu.edu.tw; metaryx.ece00g@gmail.com).

Digital Object Identifier 10.1109/TBME.2013.2252345
Aminian et al. [6] used a tri-axial accelerometer placed in a waist belt (located on the back) and a single-axis accelerometer fixed on the top of the right heel to record body accelerations during walking. Two neural networks were implemented to estimate the incline and the speed of walking. Schutz et al. [7] put a tri-axial accelerometer on the chest to measure the stride time and applied two neural network models: one for determining whether the subject walks or runs, and the other with different node interactions to estimate stride length. Song et al. [8] presented a regression method under a Gaussian process. They proposed a nonlinear, nonparametric regression framework to estimate walking speed using inertia sensors like an accelerometer and a gyroscope. He and Zhang [9] proposed the use of a portable device with a tri-axis accelerometer, and an ANN was trained to estimate the average walking speed. Kourogi and Kurata [10] observed empirically that the differences between the positive and negative peaks for vertical acceleration and forward direction have a strong correlation with walking velocity. The results from linear regression indicate that the slope is dependent on the individual person.

The methods mentioned previously require minimal knowledge of human walking but they suffer from the criticism of formal justifications. Another direction is to apply the integral relation among acceleration-velocity-position to observe the stride as well as the walking speed. The most successful method is to use a foot-mounted accelerometer and gyro to perform strapdown integration to avoid the drifting problem [11], [12]. This zero velocity updates (ZVUs) idea was also proposed in [13] using an extended Kalman filter (EKF). Combined with other sensors (such as GPS), the method is able to achieve $0.3 \%$ positioning accuracy in 3-D localization. The ZVUs are effective due to the swing-stance cycle of both legs but cannot be applied on sensors mounted on other parts of the human body.

Very few works consider the characteristics of the modelbased kinematic walking models to estimate walking speed. Bishop and Li [14] used two shank-mounted accelerometers to take advantage of the inverted pendulum-like behavior of the stance phase to estimate the walking speed. For biped walking, the inverted pendulum model is the simplest one for analysis of the kinematics or energy-spending sequences. The authors in [15] explained that human walking behavior can be modeled as an inverted pendulum, which reduces both muscle force and work demands during single support. The authors in [16] improved the inverted pendulum model by considering the effect of foot length, and this was proved by experiment result. The authors in [17] presented the advantages of the rolling-foot 


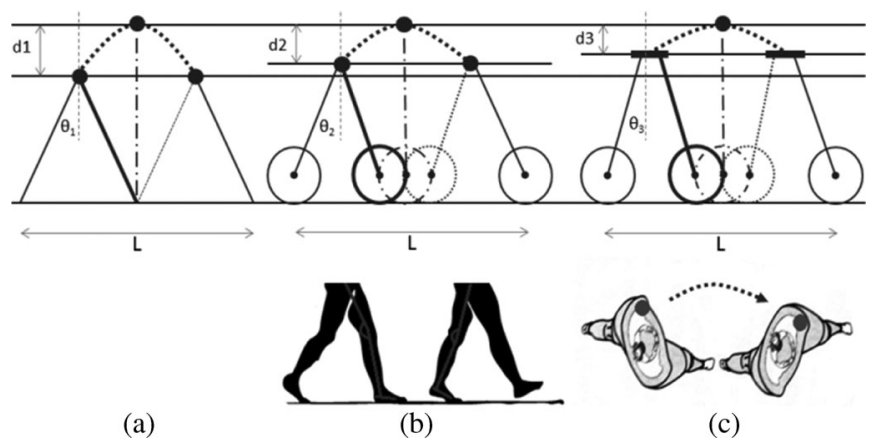

Fig. 1. [18] This series illustrates the individual and additive effects of three kinematic strategies to reduce vertical displacement of the COM: (a) illustrates the large vertical oscillation of the COM while a person walks like an inverted pendulum; (b) illustrates that further reduction of the downward displacement of the COM is achieved by rotation of the ankle and knee, and the effects are combined into the rolling-foot model; and (c) illustrates that rotation of the pelvis in the horizontal plane functionally lengthens the lower extremities and reduces the magnitude of the hip flexion-extension angle required for a given step length, thereby reducing the downward displacement of the COM, where $d_{3}<d_{2}<d_{1}, \theta_{3}<\theta_{2}<\theta_{1}$.

effect in human walking. Moreover, Neumann [18] pointed out that the vertical displacement of the COM (center of mass) is reduced to save energy during a walking cycle. That is, for a given stride length, the pure inverted pendulum that results in a large vertical displacement may not be a good model to describe walking. In reality, a human combines rolling of the ankle and knee and rotation of the waist to reduce the displacement of the COM as shown in Fig. 1.

This paper develops a kinematic walking model and proposes a method for estimating speed (in the same way as stride distance by integrating the speed). The model allows us to estimate the speed using accelerometers mounted near the COM (the center of the waist). The idea of a virtual inverted pendulum, derived from the rolling-foot model [15]-[18], is presented. Moreover, this model is improved by considering the waist rotation effect. As a result, the acceleration perpendicular to the sagittal plane is included to enhance the estimation accuracy. Interestingly, it is found out that the accelerations in the forward and vertical directions can be used to compute the radius of the rolling foot, which leads to the speed estimation. And this observation provides an explanation of the positive and negative peaks for vertical acceleration and forward direction having a strong correlation with walking velocity in [10]. The nonlinear kinematic model is estimated using an unscented Kalman Filter. Experimental results for a group of 20 persons show the effectiveness of the proposed method.

\section{Kinematic Walking Model for Speed Estimation}

\section{A. Review of Human-Walking Model}

Theoretical and experimental works on human walking have been extensively studied over the past century. A majority of works used the inverted pendulum model because it can describe the general pattern of mechanical energy fluctuations of the body during walking [19]. The COM vaulted over the rigid
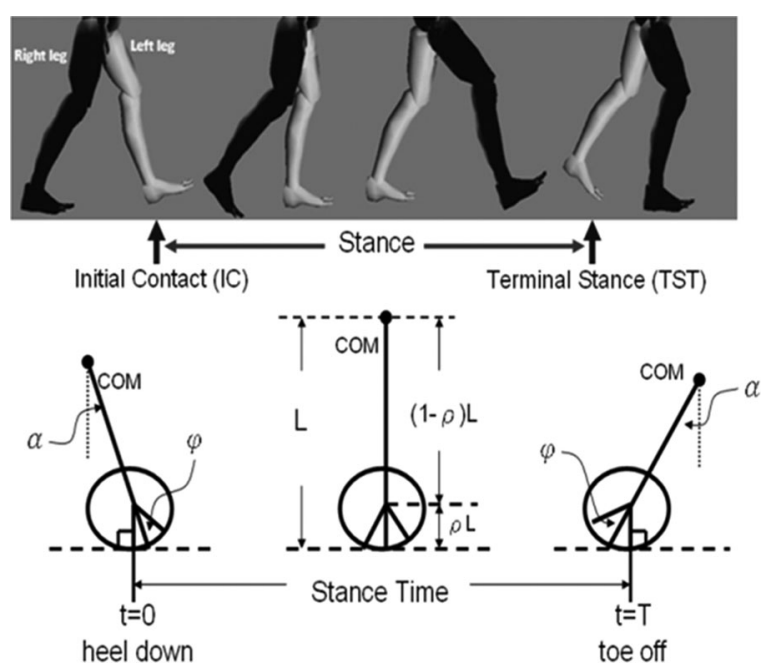

Fig. 2. Correspondence between stance and rolling-foot states.

stance limb like an inverted pendulum. This made the COM travel along a circular arc instead of along the flattened trajectory depicted by the gait theory of six determinants [15]. The flattened trajectory was proposed based on the conjecture that vertical movement of the COM is energetically costly. However, experimental results showed that humans expend more metabolic energy to voluntarily reduce vertical displacement of the COM compared to their normal gait [20]. It was also shown that the simple inverted pendulum model can be used to describe the gait transitions in terms of energy expenditure and work done on the COM [21].

Research was done to modify the vertical displacement of the COM in the perfect inverted pendulum model [16]. In [16], a constant-length virtual stance limb with a forward-translating point of force application (PFA) is assumed. It was found that the reduction of the COM vertical displacement is still unable to match the measured data. A further modification is to assume that the virtual stance limb is compressed in the stance phase. The compression is proportional to the walking speed. Another direction is to consider the rolling of the foot like a wheel during each walking step [17]. The rolling-foot model results in a modification of the inverted pendulum whereby the center of rotation should be at a distance above the point of contact with the ground. The distance was characterized as $\rho L$, where $L$ is the length of the leg and $\rho$ is a ratio $(0 \leq \rho \leq 1)$ that varies among individuals. The work in [17] shows that the minimum value of $\rho$ required to decrease the net metabolic rate is 0.3 . All of the works mentioned focused on studying the energy exchange mechanism, the force reaction on the ground, the metabolic rate, and so on. No efforts have been made to derive a kinematic model that could be utilized to estimate the walking speed through inertia sensor measurement. However, the experimental data and qualitative explanations provide basic guidelines for developing the model for estimation. In this study, the walking-speed estimator is based on the rolling-foot model of [17] as depicted in Fig. 2. 


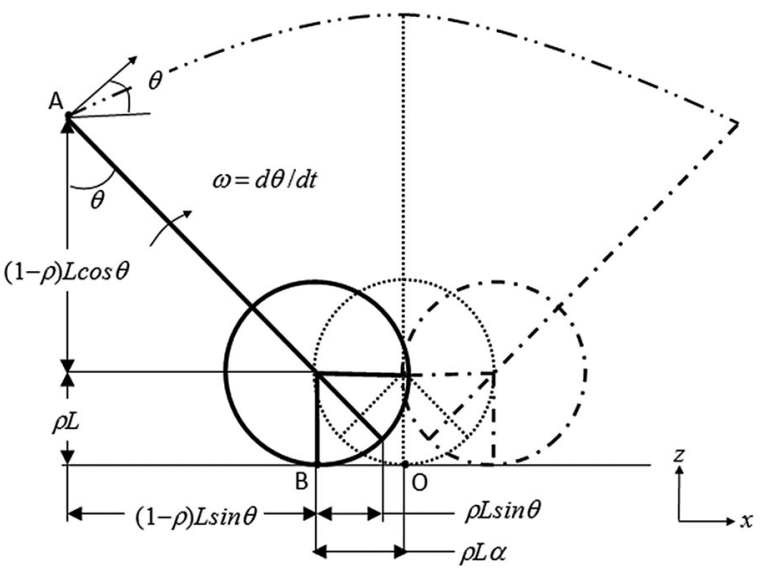

Fig. 3. Detailed rolling-foot walking model.

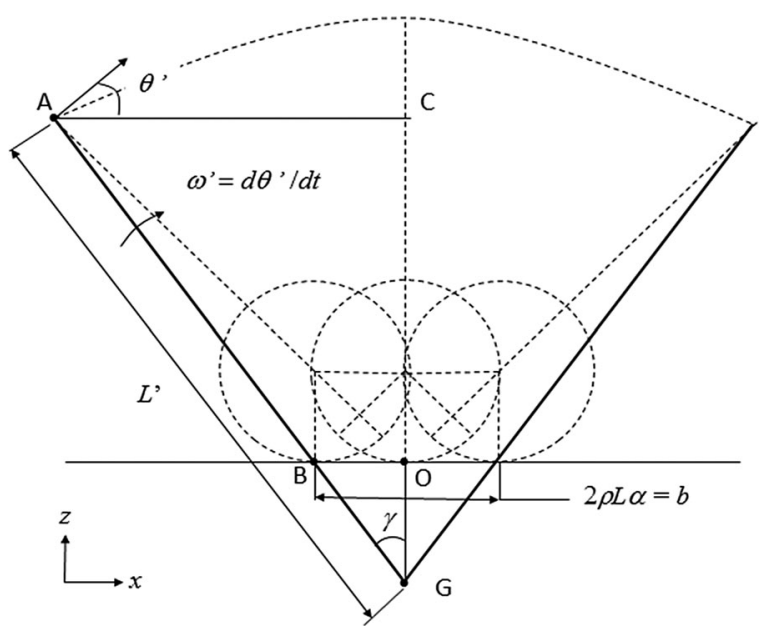

Fig. 4. Proposed virtual-center walking model during stance.

\section{B. Proposed Virtual-Center Walking Model}

The stance time $T$ defined in Fig. 2 is the duration between the initial contact (TC, the heel-down) and terminal stance (TST, toe-off). The angle $\alpha$ is the maximum swing angle of the leg and is identical for both legs. Further, it is assumed that the angle is the same for TC and TST. The leg remains stiff during the inverted pendulum swing and the rotation center is located at a distance $L$ above the ground in the $z$-axis as indicated in Fig. 3. In the sequence, the $x, y$, and $z$ coordinates are referred to as the directions perpendicular to the coronal, sagittal, and transverse planes in the human anatomical planes, respectively. The geometrical relation in Fig. 2 leads to $\alpha=\varphi$, the length of the foot is $b$, and the following relation can be established:

$$
2 \alpha \cdot \rho L=b .
$$

To facilitate the walking-speed estimation, a new model is proposed to approximate the trajectory as indicated in Fig. 4. It is assumed that during the stance stage, the leg rotates around a point located below the ground (point $\mathrm{G}$ in Fig. 4).

The COM trajectories in the $z$-direction (see Fig. 4) can be derived for the three models explained previously.

1) Inverted pendulum: $L \cos \theta, 0 \leq \theta \leq \alpha$.

2) Rolling foot: $(1-\rho) L \cos \theta+\rho L, 0 \leq \theta \leq \alpha$.

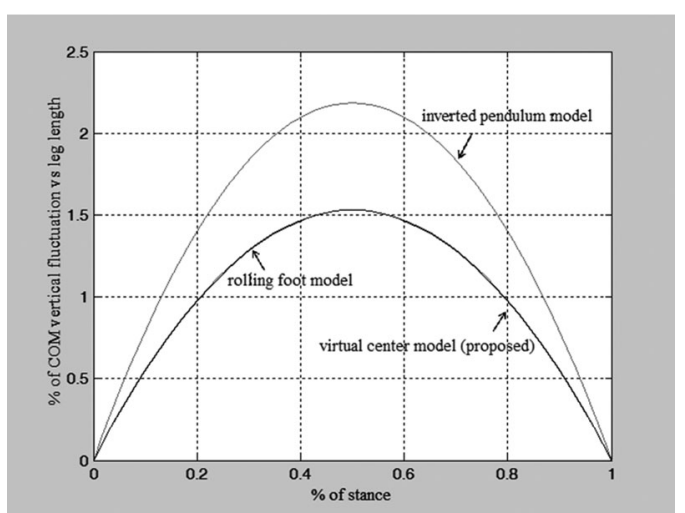

Fig. 5. Vertical fluctuation of the COM versus the leg length during stance.

3) Virtual center: $c_{1} \cos \gamma+\left(c_{1} \cos \gamma-c_{2}\right) \frac{\rho \alpha}{(1-\rho) \sin \alpha}$,

$$
0 \leq \gamma \leq \tan ^{-1}\left(\frac{(1-\rho) L \sin \alpha}{c_{2}}\right)
$$

where

$$
\begin{aligned}
& c_{1}=\sqrt{\left(1-2 \rho+2 \rho^{2}\right) L^{2}+2 \rho(1-\rho) L^{2} \cos \alpha} \\
& c_{2}=(1-\rho) L \cos \alpha+\rho L .
\end{aligned}
$$

The vertical fluctuation of the COM versus the leg length during stance is plotted for the three models in Fig. 5. The ratio $\rho$ is 0.3 as explained in [17], where the metabolic rate reaches a minimum. It is shown that the inverted pendulum has a larger fluctuation and the proposed virtual center model is close to the rolling-foot one (in Fig. 5, the rolling and proposed lines are almost the same).

\section{Parameter Estimation Using the COM Acceleration}

A method of estimating the length of the virtual leg ( $L^{\prime}$ in Fig. 4) is presented below. The estimation is based on the acceleration measurement at the COM over a complete stance cycle. Assuming that $\alpha \approx \varphi$ and $\overline{A B}+\overline{B G}=L^{\prime}$ in Fig. 4, we have

$$
\begin{aligned}
& \sqrt{(1-\rho)^{2} L^{2}+\rho^{2} L^{2}+2(1-\rho) \rho L^{2} \cos \alpha}+\frac{b}{2} \csc \gamma \\
& =L^{\prime}=L+\frac{b}{2} \cot \gamma .
\end{aligned}
$$

Therefore,

$$
\begin{aligned}
L & +\frac{b}{2} \frac{\cos \gamma-1}{\sin \gamma}=L+\frac{b}{2} \frac{-\gamma^{2} / 2 !+\gamma^{4} / 4 !-\cdots}{\gamma-\gamma^{3} / 3 !+\gamma^{5} / 5 !-\cdots} \\
& =\sqrt{(1-\rho)^{2} L^{2}+\rho^{2} L^{2}+2(1-\rho) \rho L^{2} \cos \alpha} .
\end{aligned}
$$

Approximating $\sin (\gamma)$ and $\cos (\gamma)$ by a Taylor series and keeping the first-order result, the following relation (4) is established, and $0 \leq \gamma \leq \alpha$ :

$\gamma=\frac{2 ! \times 2}{b}\left(L-\sqrt{(1-\rho)^{2} L^{2}+\rho^{2} L^{2}+2(1-\rho) \rho L^{2} \cos \alpha}\right)$.

The average tangential velocity $u$ under the rolling-foot model can be approximated by

$$
u \approx L \cdot(1-\rho) \cdot \frac{2 \alpha}{T}
$$


where $T$ is the stance duration. From (1) and Fig. 3, the COM velocity in the $x$-direction and $z$-direction can be derived as

$$
\begin{aligned}
V_{x}(t) & =u \cdot \cos (\theta(t))+\frac{b}{T} \\
& =\frac{b}{T} \cdot \frac{(1-\rho)}{\rho} \cdot \cos (\alpha-\omega t)+\frac{b}{T} \\
V_{z}(t) & =u \cdot \sin (\theta(t))=\frac{b}{T} \cdot \frac{(1-\rho)}{\rho} \cdot \sin (\alpha-\omega t)
\end{aligned}
$$

where $t=0(I C) \sim T(T S T)$. The corresponding acceleration is

$$
\begin{aligned}
& a_{x}(t)=\frac{d V_{x}(t)}{d t}=u \cdot \omega \cdot \sin (\alpha-\omega t) \\
& a_{z}(t)=\frac{d V_{z}(t)}{d t}=-u \cdot \omega \cdot \cos (\alpha-\omega t) .
\end{aligned}
$$

It is obvious that

$$
a_{x}^{2}+a_{z}^{2}=\omega^{2} u^{2} .
$$

Substituting (1) and (5) into (10) results in

$$
a_{x}^{2}+a_{z}^{2}=\left(\frac{b}{L \rho T}\right)^{2}\left(\frac{b}{T} \cdot \frac{(1-\rho)}{\rho}\right)^{2} .
$$

Lett $c=\frac{b^{4}}{L^{2} T^{4}\left(a_{x}^{2}+a_{z}^{2}\right)}$, (11) can be rewritten as the following:

$$
c(1-\rho)^{2}=\rho^{4} .
$$

Since $c$ is a positive number, (12) has only one real root located within [0,1], as shown in (13). The solution is then used to compute $\alpha$ from (1) and $\gamma$ from (4). Subsequently, the length of the virtual leg $L^{\prime}$ can be found from (2)

$$
\rho=\frac{-\sqrt{c}+\sqrt{c+4 \sqrt{c}}}{2} .
$$

\section{Proposed 3-D Walking Model}

The walking model explained previously considers the rotation of the COM in the $y$-direction which results in the fluctuation of acceleration in the $x$-direction. However, experimental data also show the rotation of the whole human body in the other two directions [18], [22]. The authors in [23] puts two 6D IMU(Inertial Measurement Unit) devices on the shoe-heels to record inertia data and describes the validation for assessment of 3-D spatial parameters of gait, which is based on the computation of 3-D foot kinematics. In particular, the ground forces during walking create torque around the $z$-direction, making the pelvis rotate around the stance leg. Consequently, the COM also moves in the $y$-direction. This phenomenon is illustrated by the top view of the walking cycles in Fig. 6. To include the COM motion in the $y$-direction, the virtual center walking model in Fig. 4 is extended to include the rotating motion of the pelvis as shown in Fig. 7.

Using the homogeneous coordinate representation, the location of the COM relative to the virtual center $G$ can be derived

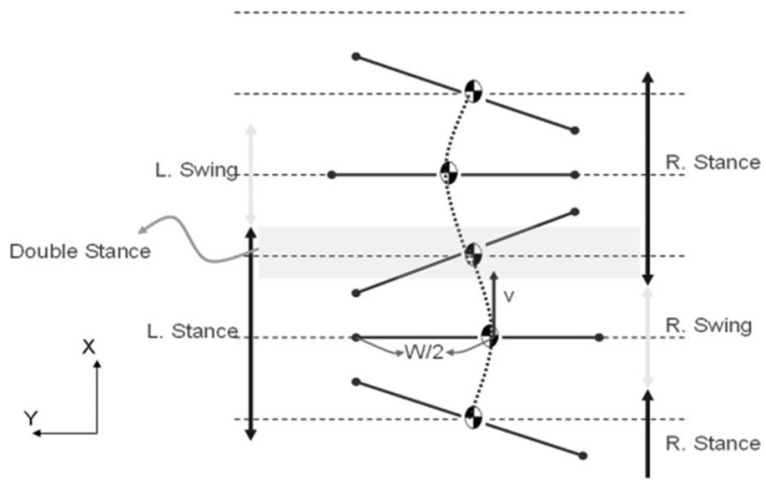

Fig. 6. Top View of the COM motion during a walking cycle.

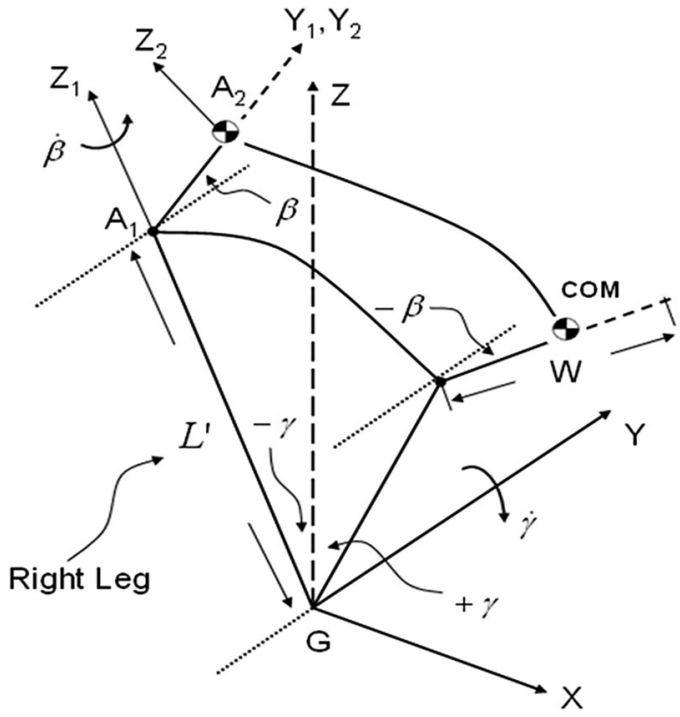

Fig. 7. Movement of the COM including the pelvis rotation ( $W=$ width of pelvis, $\beta$ : pelvis rotation angle).

as

$$
P_{\mathrm{COM}}=H_{G 1} H_{12}\left[\begin{array}{l}
0 \\
0 \\
0 \\
1
\end{array}\right]=\left[\begin{array}{c}
-\frac{W}{2} \cos (\gamma) \sin (\beta)+L^{\prime} \sin (\gamma) \\
\frac{W}{2} \cos (\beta) \\
\frac{W}{2} \sin (\gamma) \sin (\beta)+L^{\prime} \cos (\gamma) \\
1
\end{array}\right]_{G}
$$

where

$$
H_{G 1}=\left[\begin{array}{cccc}
\cos (\gamma) & 0 & \sin (\gamma) & 0 \\
0 & 1 & 0 & 0 \\
-\sin (\gamma) & 0 & \cos (\gamma) & 0 \\
0 & 0 & 0 & 1
\end{array}\right] \cdot\left[\begin{array}{cccc}
1 & 0 & 0 & 0 \\
0 & 1 & 0 & 0 \\
0 & 0 & 1 & L^{\prime} \\
0 & 0 & 0 & 1
\end{array}\right]
$$

and

$$
H_{12}=\left[\begin{array}{cccc}
\cos (\beta) & -\sin (\beta) & 0 & 0 \\
\sin (\beta) & \cos (\beta) & 0 & 0 \\
0 & 0 & 1 & 0 \\
0 & 0 & 0 & 1
\end{array}\right] \cdot\left[\begin{array}{cccc}
1 & 0 & 0 & 0 \\
0 & 1 & 0 & W / 2 \\
0 & 0 & 1 & 0 \\
0 & 0 & 0 & 1
\end{array}\right] .
$$


Taking the time derivatives of (14) and assuming that $\ddot{\beta} \approx 0$ and $\ddot{\gamma} \approx 0$, the $\mathrm{COM}$ velocity $V_{\mathrm{COM}}$ and acceleration $A_{\mathrm{COM}}$ can be computed as

$$
\begin{aligned}
& V_{\mathrm{COM}}=\frac{d}{d t} P_{\mathrm{COM}} \\
& =\left[\begin{array}{c}
\frac{W}{2} \sin (\gamma) \sin (\beta) \dot{\gamma}-\frac{W}{2} \cos (\gamma) \cos (\beta) \dot{\beta}+L^{\prime} \cos (\gamma) \dot{\gamma} \\
-\frac{W}{2} \sin (\beta) \dot{\beta} \\
\frac{W}{2} \cos (\gamma) \sin (\beta) \dot{\gamma}+\frac{W}{2} \sin (\gamma) \cos (\beta) \dot{\beta}-L^{\prime} \sin (\gamma) \dot{\gamma} \\
0
\end{array}\right]_{G}^{(15)}
\end{aligned}
$$$$
A_{\mathrm{COM}}=\frac{d}{d t} V_{\mathrm{COM}}
$$

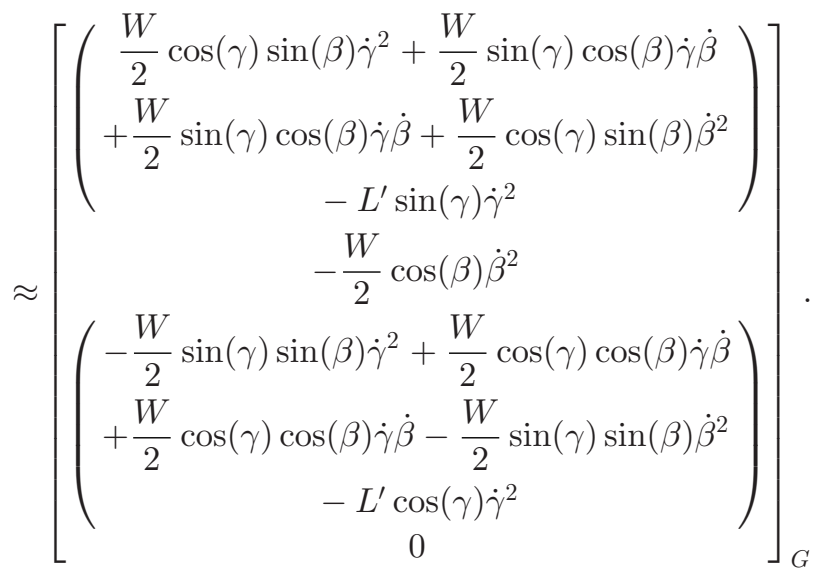

In normal walking, the ranges of $\beta$ and $\gamma$ are within $\pm 0.17 \mathrm{rad}$. Therefore, a small-angle model is adopted by making $\sin (\beta) \approx$ $\beta, \cos (\beta) \approx 1, \sin (\gamma) \approx \gamma, \cos (\gamma) \approx 1$. The average COM velocity is then computed by integrating $\mathrm{V}_{\mathrm{COM}}$ with respect to $\beta$ and $\gamma$ over a stance period

$$
\begin{aligned}
\bar{V}_{\mathrm{COM}} & =\frac{1}{2 \beta} \frac{1}{2 \gamma} \int_{-\beta}^{\beta} \int_{-\gamma}^{\gamma} V_{\mathrm{COM}}(\beta, \gamma) \cdot d \gamma \cdot d \beta \\
& \approx\left[\begin{array}{c}
-\frac{W}{2} \dot{\beta}+L^{\prime} \dot{\gamma} \\
0 \\
0 \\
0
\end{array}\right]_{G}=\left[\begin{array}{c}
v+u \\
0 \\
0 \\
0
\end{array}\right]_{G}
\end{aligned}
$$

where $v=-\frac{W}{2} \dot{\beta}$ and $u=L^{\prime} \dot{\gamma}$. The acceleration of (16) can also be simplified into

$$
\begin{aligned}
A_{\mathrm{COM}} \approx & {\left[\begin{array}{c}
\frac{W}{2} \beta \dot{\gamma}^{2}+W \gamma \dot{\gamma} \dot{\beta}+\frac{W}{2} \beta \dot{\beta}^{2}-L^{\prime} \gamma \dot{\gamma}^{2} \\
-\frac{W}{2} \dot{\beta}^{2} \\
-\frac{W}{2} \gamma \beta \dot{\gamma}^{2}+W \dot{\gamma} \dot{\beta}-\frac{W}{2} \gamma \beta \dot{\beta}^{2}-L^{\prime} \dot{\gamma}^{2} \\
0
\end{array}\right]_{G} } \\
= & {\left[\begin{array}{c}
a_{x} \\
a_{y} \\
a_{z} \\
0
\end{array}\right]_{G} . }
\end{aligned}
$$

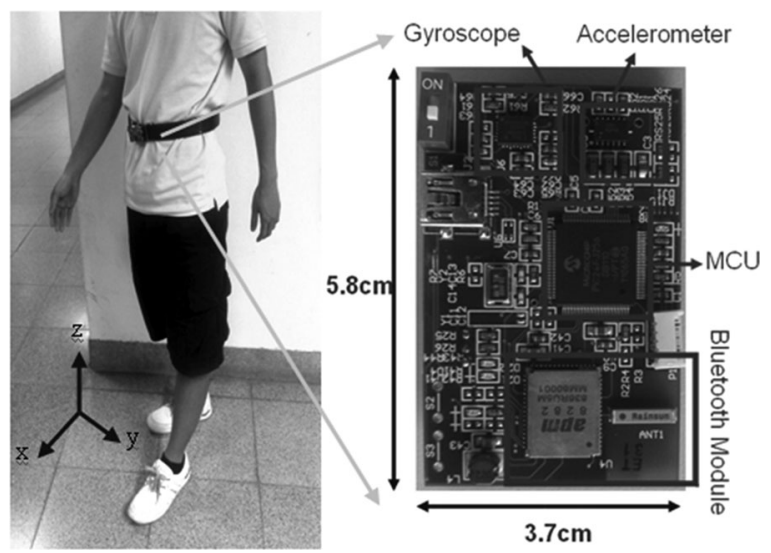

Fig. 8. Tri-axial accelerometer device and mounting position.

It is obvious that

$$
\left|a_{y}\right|=\frac{2 v^{2}}{W}
$$

because $\beta, \gamma \approx 0$, and $\sin ^{2} \beta+\cos ^{2} \beta \approx 1+\beta^{2} \approx 1+\gamma^{2} \approx$ 1. Neglecting all the terms of the sixth order and higher of the angle $\beta$ and $\gamma$, the following relation can be established:

$$
\sqrt{a_{x}^{2}+a_{z}^{2}}=\frac{u^{2}+u \cdot 2 v}{L^{\prime}} .
$$

Equations (17), (19), and (20) form the basic formula for estimating the walking speed as described in the following section.

\section{Walking SPEEd Estimation AlgORITHM}

The device for measuring the walking speed is a tri-axial accelerometer mounted at the center of the waist (see Fig. 8). It is easy to install the device by clipping it on a belt. The acceleration measurements (as depicted by Fig. 8) are preprocessed by a standard bias and gravity estimation algorithm to minimize their influences. Meanwhile, a low-pass filter (cut-off frequency at $3 \mathrm{~Hz}$ ) is implemented to remove high-frequency noise that is irrelevant to the human body motion. It is assumed that the measurements are close to the COM acceleration signals even though it is not mounted exactly at the COM position. This will induce some errors and will be discussed in the experiments.

Equations (19) and (20) can be rewritten into a state-space formula to estimate both $u$ and $v$ as the following:

$$
\begin{aligned}
\mathbf{x}(k+1) & =\mathbf{x}(k)+\mathbf{q}(k) \\
\mathbf{y}(k) & =\mathbf{H}(\mathbf{x}(k))+\mathbf{d}(k)
\end{aligned}
$$

where $\mathbf{x}(k)=\left[\begin{array}{ll}u(k) & v(k)\end{array}\right]^{T}$ is the parameter vector to be estimated and

$$
\begin{aligned}
& \mathbf{y}(k)=\left[\begin{array}{ll}
\sqrt{a_{x}^{2}(k)+a_{z}^{2}(k)} & \left.\left|a_{y}(k)\right|\right]^{T}
\end{array}\right. \\
& \mathbf{H}(\mathbf{x}(k))=\left[\begin{array}{ll}
\frac{u^{2}(k)+u(k) \cdot 2 v(k)}{L^{\prime}} & \frac{v^{2}(k)}{W / 2}
\end{array}\right]^{T}
\end{aligned}
$$

$\mathbf{q}(k)$ and $\mathbf{d}(k)$ are uncorrelated zero-mean Gaussian noise vectors with covariance matrices $\mathbf{Q}$ and $\mathbf{R}$, respectively. The parameter $L^{\prime}$ is obtained from (2) for each sample. Equations (19) and (20) can be estimated using the unscented Kalman filter due to 
TABLE I

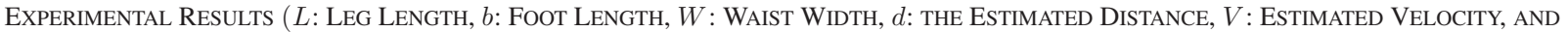
$\operatorname{error}(\%)=(d-38) / 38 \times 100 \%)$

\begin{tabular}{|c|c|c|c|c|c|c|c|c|c|c|c|c|c|c|}
\hline \multirow{3}{*}{ No. } & \multicolumn{14}{|c|}{ User information and results } \\
\hline & \multicolumn{2}{|c|}{$L(m)$} & \multicolumn{2}{|c|}{$b(m)$} & \multicolumn{2}{|c|}{$W(m)$} & \multicolumn{2}{|c|}{$P$} & \multicolumn{2}{|c|}{$d(m)$} & \multicolumn{2}{|c|}{$V(m / s)$} & \multicolumn{2}{|c|}{ error(\%) } \\
\hline & Nominal & Calibrated & Nominal & Calibrated & Nominal & Calibrated & $\begin{array}{l}\text { Before } \\
\text { Calibr. }\end{array}$ & $\begin{array}{c}\text { After } \\
\text { Calibr. }\end{array}$ & $\begin{array}{l}\text { Before } \\
\text { Calibr. }\end{array}$ & $\begin{array}{c}\text { After } \\
\text { Calibr. }\end{array}$ & $\begin{array}{l}\text { Before } \\
\text { Calibr. }\end{array}$ & $\begin{array}{c}\text { After } \\
\text { Calibr. }\end{array}$ & $\begin{array}{l}\text { Before } \\
\text { Calibr. }\end{array}$ & $\begin{array}{c}\text { After } \\
\text { Calibr. }\end{array}$ \\
\hline 1 & 1.00 & 0.95 & 0.20 & 0.20 & 0.40 & 0.36 & 0.28 & 0.29 & 38.94 & 38.05 & 1.42 & 1.39 & 2.47 & 0.14 \\
\hline 2 & 1.02 & 0.97 & 0.20 & 0.18 & 0.40 & 0.38 & 0.31 & 0.29 & 39.43 & 37.95 & 1.27 & 1.26 & 3.77 & -0.14 \\
\hline 3 & 1.05 & 0.95 & 0.21 & 0.22 & 0.40 & 0.36 & 0.27 & 0.29 & 39.45 & 38.05 & 1.55 & 1.49 & 3.81 & 0.14 \\
\hline 4 & 1.05 & 1.00 & 0.23 & 0.21 & 0.43 & 0.41 & 0.31 & 0.29 & 39.09 & 37.73 & 1.45 & 1.40 & 2.88 & -0.71 \\
\hline 5 & 1.07 & 1.07 & 0.20 & 0.18 & 0.40 & 0.38 & 0.32 & 0.29 & 38.20 & 37.52 & 1.31 & 1.29 & 0.52 & -1.26 \\
\hline 6 & 0.91 & 0.86 & 0.18 & 0.16 & 0.40 & 0.36 & 0.31 & 0.29 & 40.03 & 38.32 & 1.37 & 1.31 & 5.34 & 0.84 \\
\hline 7 & 1.03 & 0.98 & 0.22 & 0.22 & 0.40 & 0.44 & 0.28 & 0.29 & 38.82 & 38.09 & 1.54 & 1.51 & 2.16 & 0.24 \\
\hline 8 & 1.02 & 1.02 & 0.20 & 0.18 & 0.38 & 0.36 & 0.32 & 0.29 & 38.70 & 37.97 & 1.24 & 1.22 & 1.83 & -0.07 \\
\hline 9 & 1.02 & 1.06 & 0.20 & 0.18 & 0.40 & 0.36 & 0.31 & 0.29 & 37.65 & 37.57 & 1.25 & 1.25 & -0.93 & -1.12 \\
\hline 10 & 1.02 & 1.11 & 0.18 & 0.17 & 0.38 & 0.40 & 0.31 & 0.29 & 37.11 & 38.21 & 1.17 & 1.20 & -2.35 & 0.56 \\
\hline 11 & 0.93 & 0.98 & 0.20 & 0.20 & 0.36 & 0.38 & 0.31 & 0.30 & 37.52 & 38.37 & 1.32 & 1.35 & -1.23 & 0.96 \\
\hline 12 & 0.91 & 0.81 & 0.17 & 0.15 & 0.30 & 0.33 & 0.31 & 0.29 & 40.12 & 37.60 & 1.16 & 1.09 & 5.58 & -1.04 \\
\hline 13 & 0.93 & 0.88 & 0.17 & 0.15 & 0.31 & 0.29 & 0.31 & 0.29 & 40.01 & 38.32 & 0.97 & 0.93 & 5.29 & 0.84 \\
\hline 14 & 0.96 & 0.96 & 0.18 & 0.16 & 0.36 & 0.32 & 0.33 & 0.30 & 38.73 & 37.85 & 1.25 & 1.23 & 1.93 & -0.39 \\
\hline 15 & 0.82 & 0.77 & 0.16 & 0.15 & 0.29 & 0.27 & 0.31 & 0.29 & 39.85 & 37.99 & 1.01 & 0.97 & 4.88 & -0.14 \\
\hline 16 & 0.96 & 0.91 & 0.20 & 0.18 & 0.35 & 0.38 & 0.31 & 0.29 & 39.31 & 37.85 & 1.26 & 1.21 & 3.46 & -0.39 \\
\hline 17 & 1.00 & 0.95 & 0.19 & 0.16 & 0.31 & 0.31 & 0.33 & 0.29 & 40.23 & 38.29 & 1.14 & 1.09 & 5.87 & 0.78 \\
\hline 18 & 0.95 & 0.90 & 0.19 & 0.18 & 0.34 & 0.36 & 0.31 & 0.30 & 39.40 & 38.30 & 1.18 & 1.15 & 3.69 & 0.78 \\
\hline 19 & 1.00 & 0.95 & 0.21 & 0.19 & 0.32 & 0.29 & 0.30 & 0.28 & 39.53 & 38.01 & 1.36 & 1.30 & 4.02 & 0.02 \\
\hline 20 & 0.95 & 0.86 & 0.18 & 0.16 & 0.31 & 0.33 & 0.30 & 0.29 & 39.98 & 37.58 & 1.17 & 1.10 & 5.22 & -1.11 \\
\hline
\end{tabular}

its good performance in dealing with second-order nonlinearity.

The computation procedure is listed below.

1) Time update and sigma point computation:

$$
\begin{aligned}
\hat{\mathbf{x}}^{-}(k)= & \hat{\mathbf{x}}(k-1) \\
\mathbf{P}_{\mathbf{x}(\mathbf{k})}^{-}= & \mathbf{P}_{\mathbf{x}(\mathbf{k}-1)}+\mathbf{Q} \\
\mathbf{X}_{\mathbf{k} \mid \mathbf{k}-\mathbf{1}}= & {\left[\hat{\mathbf{x}}^{-}(k) \hat{\mathbf{x}}^{-}(k)+\gamma_{\mathrm{UKF}} \sqrt{\mathbf{P}_{\mathbf{x}(\mathbf{k})}^{-}}\right.} \\
& \left.\hat{\mathbf{x}}^{-}(k)-\gamma_{\mathrm{UKF}} \sqrt{\mathbf{P}_{\mathbf{x}(\mathbf{k})}^{-}}\right] \\
\mathbf{D}_{\mathbf{k} \mid \mathbf{k}-\mathbf{1}}= & \mathbf{H}\left[X_{\mathbf{k} \mid \mathbf{k}-\mathbf{1}}\right] \\
\hat{\mathbf{d}}(k)= & \sum_{\mathbf{i}=\mathbf{0}}^{\mathbf{L}} \mathbf{W}_{\mathbf{i}}^{(\mathbf{m})} \mathbf{D}_{i, \mathbf{k} \mid \mathbf{k}-\mathbf{1}} .
\end{aligned}
$$

2) Measurement update:

$$
\begin{aligned}
& \mathbf{P}_{\tilde{\mathbf{d}}(k) \tilde{\mathbf{d}}(k)}=\sum_{\mathbf{i}=\mathbf{0}}^{2 \mathbf{L}} \mathbf{W}_{\mathbf{i}}^{(c)}\left[\mathbf{D}_{i, \mathbf{k} \mid \mathbf{k}-\mathbf{1}}-\hat{\mathbf{d}}(k)\right] \\
& \cdot\left[\mathbf{D}_{i, \mathbf{k} \mid \mathbf{k}-\mathbf{1}}-\hat{\mathbf{d}}(k)\right]^{T}+\mathbf{R} \\
& \mathbf{P}_{\mathbf{x}(k) \mathbf{d}(k)}=\sum_{\mathbf{i}=\mathbf{0}}^{\mathbf{2} \mathbf{L}} \mathbf{W}_{\mathbf{i}}^{(c)}\left[\mathbf{X}_{i, \mathbf{k} \mid \mathbf{k}-\mathbf{1}}-\mathbf{x}(k)\right] \\
& \cdot\left[\mathbf{D}_{i, \mathbf{k} \mid \mathbf{k}-\mathbf{1}}-\hat{\mathbf{d}}(k)\right]^{T} \\
& \mathbf{K}(k)=\mathbf{P}_{\mathbf{x}(k) \mathbf{d}(k)}\left(\mathbf{P}_{\tilde{\mathbf{d}}(k) \tilde{\mathbf{d}}(k)}\right)^{-1} \\
& \hat{\mathbf{x}}(k)=\hat{\mathbf{x}}^{-}(k)+\mathbf{K}(k) \cdot(\mathbf{d}(k)-\hat{\mathbf{d}}(k)) \\
& \mathbf{P}_{\mathbf{x}(\mathbf{k})}=\mathbf{P}_{\mathbf{x}(\mathbf{k})}^{-}-\mathbf{K}(k) \mathbf{P}_{\tilde{\mathbf{d}}(k) \tilde{\mathbf{d}}(k)} \mathbf{K}(k)^{T} .
\end{aligned}
$$

\section{EXPERIMENTAL RESULTS}

To verify the proposed algorithm, a group of 20 persons is asked to walk along a straight line four times with the device mounted on their waists. The walking distance is $38 \mathrm{~m}$ and the ground truth is obtained as the following. At the starting (ending) location, an external infrared sensor is installed to detect the passage of the human. The passage signals are transmitted to the measurement device instantaneously as time stamps for verification. In other words, the distance integrated from the estimated average walking speed between the two time stamps is compared with the actual distance of $38 \mathrm{~m}$. The participants were asked to walk freely at their own pace under a normal situation. The initial conditions and parameter selections of the unscented Kalman filter are shown in the Appendix. A group of 20 persons was tested to validate the proposed method. Nos. 1 to 10 are male, and the rest are female. The leg length $L$, foot length $b$, and waist size $W$ are measured manually as shown in Table I (under the "Nominal" column). Without calibrating the measurement errors, the proposed method yields an absolute average error of $3.36 \%$ and standard deviation of $2.33 \%$ (the "Before Calibr." column of Table I). With respect to the measurement errors, the results indicate that our method is still competitive in relation to existing works as listed in Table II.

To further enhance the performance, the test subjects were asked to walk four times, from which three records were used for calibration and the fourth to compute the accuracy. The calibration procedure was conducted by an exhaustive search of the optimal speed estimation error in the parameter space $(L, b$, and $W$ ) limited by $\pm 10 \%$ of the associated nominal values. As an example, Fig. 9 illustrates the distribution of the sum of mean square errors under the variation of $L$ and $b$ with a fixed $W$ for one of the test subjects. The minimum error occurs at $L=1.06$, $b=0.18$, and $W=0.36$. After calibration, the absolute average 
TABLE II

COMPARISON WITH EXISTING METHODS

\begin{tabular}{|c|c|c|c|c|c|c|c|}
\hline Ref. & \multicolumn{2}{|c|}{ Proposed } & [7] & [8] & [9] & {$[14] /[29]$} & [11] \\
\hline Year & \multicolumn{2}{|c|}{2012} & 2002 & 2007 & 2011 & 2010 & 2005 \\
\hline Sensor & \multicolumn{2}{|c|}{ Acc } & Acc & Acc & Acc & Acc \& Gyro & IMU \\
\hline Method & \multicolumn{2}{|c|}{$* * \mathrm{KM}$} & Statistics & $* \mathrm{NN}$ & $* \mathrm{NN}$ & $* * \mathrm{KM}$ & $* * \mathrm{KM}$ \\
\hline Location & \multicolumn{2}{|c|}{ Waist } & body & chest & pocket & shank & shoes \\
\hline \multirow[t]{2}{*}{ Err. Mean } & $\begin{array}{l}\text { Before } \\
\text { Calibr. }\end{array}$ & $\begin{array}{c}\text { After } \\
\text { Calibr. }\end{array}$ & \multirow[t]{2}{*}{$0.51 \%$} & \multirow[t]{2}{*}{$9.57 \%$} & \multirow[t]{2}{*}{$3.04 \%$} & \multirow[t]{2}{*}{$-17 \%$} & \multirow[t]{2}{*}{$0.3 \%$} \\
\hline & $3.36 \%$ & $0.58 \%$ & & & & & \\
\hline Err. Std & $2.33 \%$ & $0.72 \%$ & $20 \%$ & - & $4.1 \%$ & $8 \%[14] / 4 \%[29]$ & - \\
\hline Exp. No. & \multicolumn{2}{|c|}{ Test 20} & $\begin{array}{c}\text { Train } 50 \\
\text { Test } 6\end{array}$ & $\begin{array}{c}\text { Train } 15 \\
\text { Test } 2\end{array}$ & $\begin{array}{c}\text { Train } 25 \\
\text { Test } 10\end{array}$ & $18 /-$ & 1 \\
\hline
\end{tabular}

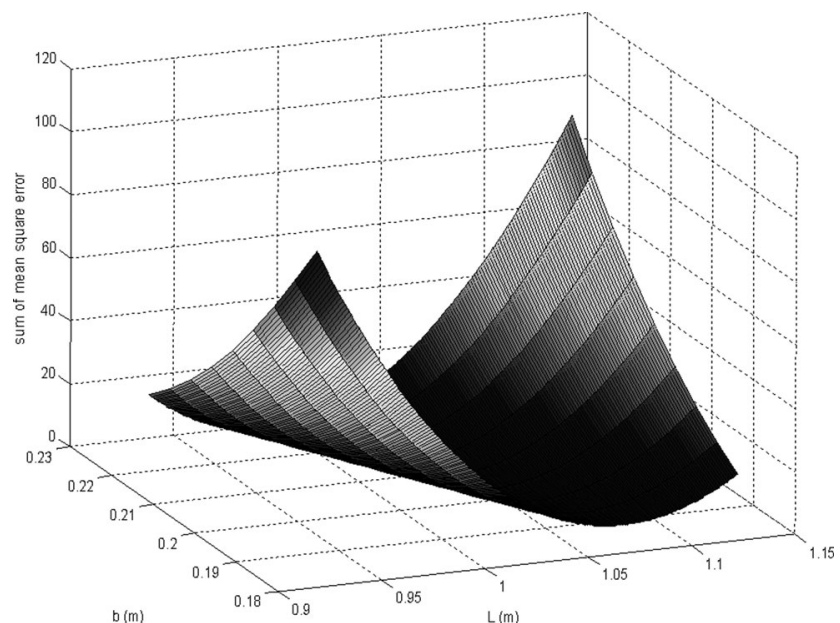

Fig. 9. Distribution between sum of the square error mean and variation of $L$ and $b$ with a fixed $W$ of subject No. 9 in Table I.
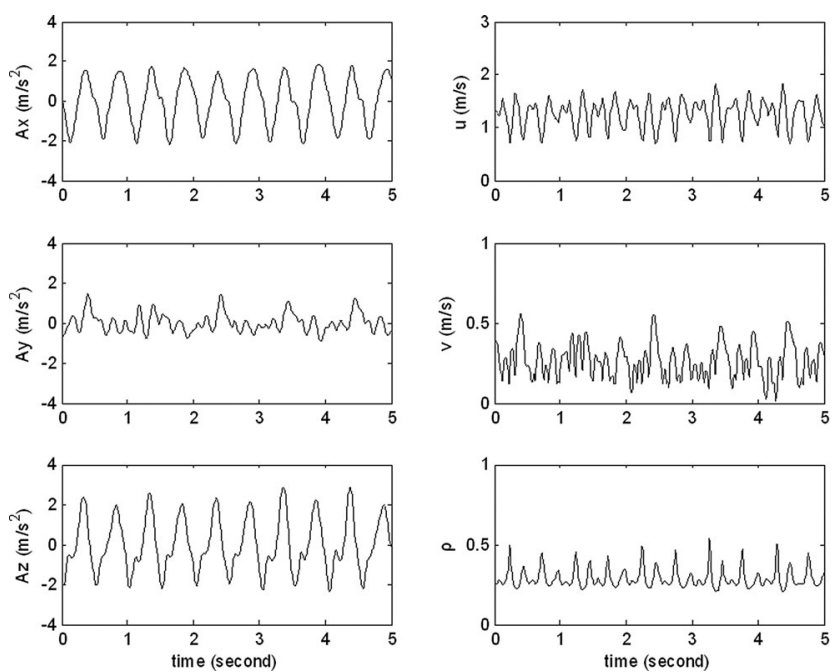

Fig. 10. Experimental data of subject No. 7 whose means of $u$ and $v$ are 1.25 and $0.26 \mathrm{~m} / \mathrm{s}$, with variances of 0.07 and 0.01 , respectively. The mean and variance of $\rho$ are 0.2865 and 0.003 . The estimated velocity $V=u+v=1.51$, and the other user parameters are shown in Table I. (Top left to bottom left: $A_{x}, A_{y}, A_{z}$; top right to bottom right: estimated parameters $u, v$, and $\rho$.)

error is reduced to $0.58 \%$ and the standard deviation to $0.72 \%$. The absolute average errors for males and females are $0.52 \%$ and $0.65 \%$, while the absolute standard deviations are $0.44 \%$ and $0.38 \%$, respectively. From the comparison in Table II, the
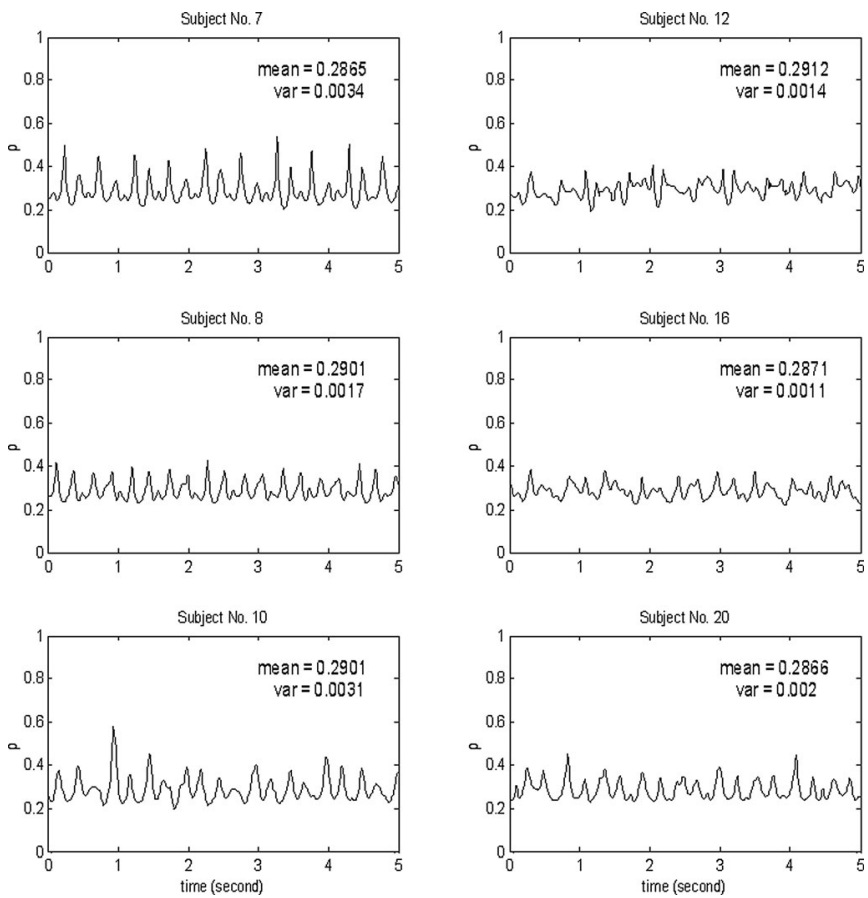

Fig. 11. $\rho$ values of six different subjects in the normal walking case. The subjects shown on the left are male cases and those on the right are female cases. The walking speeds are as follows: No. 7: $1.51 \mathrm{~m} / \mathrm{s} ;$ No. $8: 1.22 \mathrm{~m} / \mathrm{s}$; No. 10: 1.20 m/s; No. 12: 1.09 m/s; No. 16: $1.21 \mathrm{~m} / \mathrm{s}$; and No. 20: $1.1 \mathrm{~m} / \mathrm{s}$.

proposed method after calibration outperforms all the previous methods including the one using kinematic models.

The acceleration signal and estimated parameters of subject No. 7 are shown in Fig. 10. The walking speed estimation is equal to $u$ plus $v$. In this case, the means of $u$ and $v$ are 1.25 and $0.26 \mathrm{~m} / \mathrm{s}$, and the variances of $u$ and $v$ are 0.07 and 0.01 , respectively. Note that the speed $v$, estimated from the rotation of the waist, contributes approximately $17 \%$ of the total speed.

Fig. 11 shows the value of $\rho$ [see (13)] of six different subjects under normal walking. The average values for all cases are approximately 0.29 , which is close to the observation in [17] for minimum metabolic rate. However, the value of $\rho$ does not remain constant during the entire walking cycle. Some of the data are deliberately selected to have approximately the same velocity, such as Nos. 8 and 10 with a velocity of $1.2 \mathrm{~m} / \mathrm{s}$ and Nos. 12 and 20 with a velocity of $1.1 \mathrm{~m} / \mathrm{s}$. But the patterns of $\rho$ are quite different. Despite the accuracy of the gait speed 

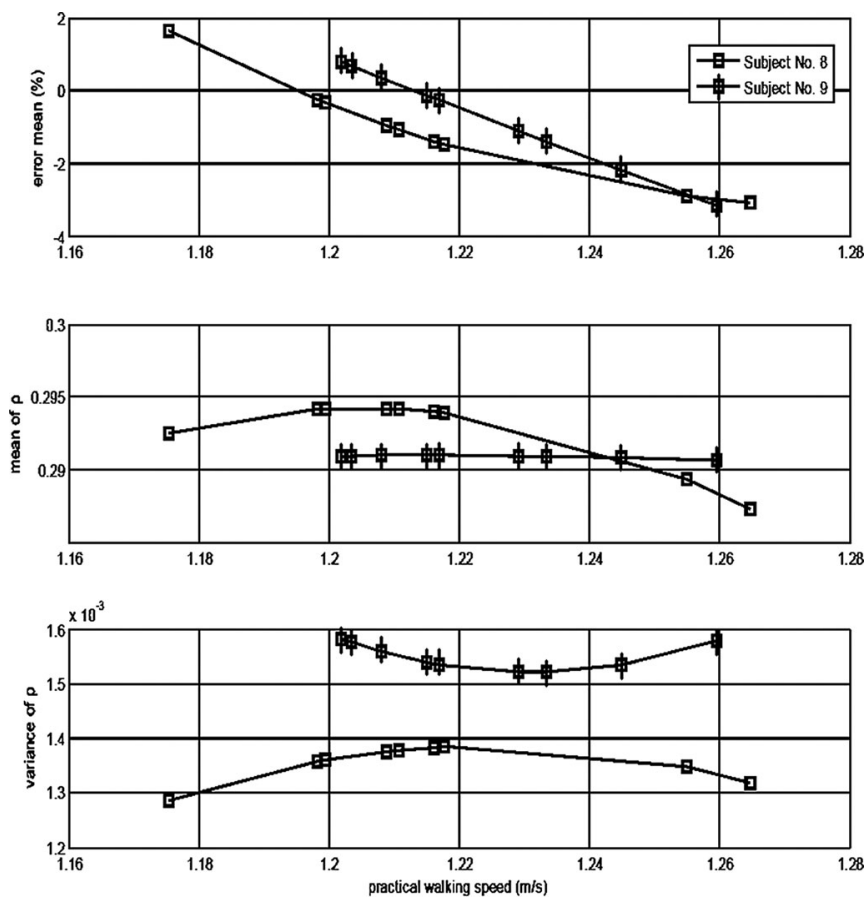

Fig. 12. Normal walking repeated nine times by two subjects; from top to bottom, the plots are the second regression of the mean error (\%), mean of $\rho$, and variance of $\rho$ versus practical walking speed $(\mathrm{m} / \mathrm{s})$.

estimation, it is still too early to say that the pattern may reflect distinct gait characteristics for each individual. This could lead to interesting observations and is currently under investigation.

Two test subjects were asked to walk at lower and higher speeds. Fig. 12 depicts the relation among different walking speeds and associated estimation errors. The error is increased when the speed is away from the normal range. Note that the variation of the mean and standard deviation of $\rho$ is very small (within the range of 0.01 and 0.0001 ). This indicates that adjusting the rolling-foot radius cannot describe the walking behavior well at lower and higher speeds. Further, investigation is underway to consider combinations of different gait patterns for a more accurate prediction.

\section{CONCLUSION}

A human-walking speed estimation algorithm is presented which uses only acceleration signals at the center of the waist. The algorithm is based on a kinematic model which combines the inverted pendulum model and the rolling-foot behavior into kinematic equations. In addition, the effect of waist rotation is also considered to achieve a more accurate estimation, and this extends the walking model into a three conjunction rod structure to describe the walking behavior approximating the practical case. The advantage of the proposed method, compared with learning methods or statistical regression, is that a training procedure is not needed. Experimental results from 20 subjects show the effectiveness of the proposed method in the normal walking-speed range, where a $0.58 \%$ mean error and $0.72 \%$ standard deviation are achieved. The result also confirms that the average ratio of the rolling-foot radius to the leg length achieves the minimum metabolic rate. However, variations of the ratio during a walking cycle reveal different patterns for different individuals. Modifications of the proposed method to extend the speed-estimation range are currently under investigation.

\section{APPENDIX \\ INITIAL CONDITIONS AND PARAMETERS OF UKF}

The initial conditions and parameters chosen for the unscented Kalman filter are as follows. The state initial conditions are set to zero. The measurement covariance matrix is set according to the standard deviation of accelerometer signals in the stationary condition. The process noise covariance matrix has equal weight for both states and the scale is equivalent to the measurement covariance. In this way, the state update will follow the measurement closely as explained and other parameters are selected following the work in [27] and [28].

$$
\begin{aligned}
& \hat{\mathbf{x}}(0)=E[\mathbf{x}]=\left[\begin{array}{ll}
0 & 0
\end{array}\right]^{T} \\
& P_{\mathbf{x}(0)}=E\left[(\mathbf{x}-\hat{\mathbf{x}}(0))(\mathbf{x}-\hat{\mathbf{x}}(0))^{T}\right]=\operatorname{diag}(0.1) \\
& \gamma_{\mathrm{UKF}}=\sqrt{L_{\mathrm{UKF}}+\lambda_{\mathrm{UKF}}} \\
& \lambda_{\mathrm{UKF}}=\alpha_{\mathrm{UKF}}^{2}\left(L_{\mathrm{UKF}}+\kappa\right)-L_{\mathrm{UKF}} \\
& \beta_{\mathrm{UKF}}=2 \\
& \alpha_{\mathrm{UKF}}=1 e-3 \\
& \kappa=0 \text { or } 3-L_{\mathrm{UKF}} \\
& L_{\mathrm{UKF}}=\text { dimension of the state }=2 \\
& \mathbf{Q}=\text { process noise covariance }=\left[\begin{array}{cc}
0.1 & 0 \\
0 & 0.1
\end{array}\right] \\
& \mathbf{R}=\text { measurement noise covariance }=\left[\begin{array}{cc}
0.2 & 0 \\
0 & 0.1
\end{array}\right] \\
& W_{0}^{(m)}=\frac{\lambda_{\mathrm{UKF}}}{L_{\mathrm{UKF}}+\lambda_{\mathrm{UKF}}} \\
& W_{0}^{(c)}=\frac{\lambda_{\mathrm{UKF}}}{L_{\mathrm{UKF}}+\lambda_{\mathrm{UKF}}}+\left(1-\alpha_{\mathrm{UKF}}^{2}+\beta_{\mathrm{UKF}}\right) \\
& W_{i}^{(m)}=W_{i}^{(c)}=\frac{1}{2\left(L_{\mathrm{UKF}}+\lambda_{\mathrm{UKF}}\right)}, i=1,2, \ldots, 2 L_{\mathrm{UKF}}
\end{aligned}
$$

\section{REFERENCES}

[1] J. J. Kavanagh and H. B. Menz, "Accelerometry: A technique for quantifying movement patterns during walking," Gait Posture, vol. 28, pp. 1-15, 2008.

[2] A. Godfrey, R. Conway, D. Meagher, and G. ÓLaighin, "Direct measurement of human movement by accelerometry," Med. Eng. Phys., vol. 30, pp. 1364-1386, 2008.

[3] S. J. Preece, J. Y. Goulermas, L. P. J. Kenney, D. Howard, K. Meijer, and R. Crompton, "Activity identification using body-mounted sensors-A review of classification techniques," Physiol. Meas., vol. 30, pp. R1-R33, 2009.

[4] J. A. Hesch and S. I. Roumeliotis, "An indoor localization aid for the visually impaired," in Proc. IEEE Int. Conf. Rob. Autom., Apr. 10-14, 2007, pp. 3545-3551.

[5] D. Alvarez, R. C. Gonzalez, A. Lopez, and J. C. Alvarez, "Comparison of step length estimators from wearable accelerometer devices," in Proc. 28th Annu. Int. Conf. IEEE Eng. Med. Biol. Soc., New York, NY, USA, Aug. 2006, pp. 5964-5967. 
[6] K. Aminian, P. Robert, E. Quier, and Y. Schutz, "Estimation of speed and incline of walking using neural network," IEEE Trans. Instrum. Meas., vol. 44, no. 3, pp. 743-746, Jun. 1995.

[7] Y. Schutz, S. Weinsier, P. Terrier, and D. Durrer, "A new accelerometric method to assess the daily walking practice," Int. J Obes. Relat. Metab. Disord., vol. 26, pp. 111-118, 2002.

[8] Y. Song, S. Shin, S. Kim, D. Lee, and K. H. Lee, "Speed estimation from a tri-axial accelerometer using neural networks," in Proc. 29th Annu. Int. Conf. IEEE EMBS, 2007, pp. 3224-3227.

[9] Z. He and W. Zhang, "Estimation of walking speed using accelerometer and artificial neural networks," Commun. Comput. Inf. Sci., vol. 159, no. 1, pp. 42-47, 2011.

[10] M. Kourogi and T. Kurata, "Personal positioning based on walking locomotion analysis with self-contained sensors and a wearable camera," in Proc. Int. Society Mag. Resonance, Tokyo, Japan, 2003, pp. 103-112.

[11] A. M. Sabatini, C. Martelloni, S. Scapellato, and F. Cavallo, "Assessment of walking features from foot inertial sensing," IEEE Trans. Biomed. Eng., vol. 52, no. 3, pp. 486-494, Mar. 2005.

[12] F. Cavallo, A. M. Sabatini, and V. Genovese, "A step toward GPS/INS personal navigation systems: Real-time assessment of gait by foot inertial sensing," in Proc. IEEE/RSJ Int. Conf. Intell. Rob. Syst., Aug. 2-6, 2005, pp. 1187-1191.

[13] E. Foxlin, "Pedestrian tracking with shoe-mounted inertial sensors," IEEE Comput. Graph. Appl., vol. 25, no. 6, pp. 38-46, Nov./Dec. 2005.

[14] E. Bishop and Q. Li, "Walking speed estimation using shank-mounted accelerometers," in Proc. IEEE Int. Conf. Rob. Autom., Anchorage, AK, USA, May 2010, pp. 5096-5101.

[15] S. A. Gard and D. S. Childress, "What determines the vertical displacement of the body during normal walking?," J. Prosthet. Orthot., vol. 13, pp. 64-67, 2001.

[16] C. R. Lee and C. T. Farley, "Determinants of the center of mass trajectory in human walking and running," J. Exp. Biol., vol. 201, pp. 2935-2944, 1998

[17] P. G. Adamczyk, S. H. Collins, and A. D. Kuo, "The advantages of a rolling foot in human walking," J. Exp. Biol., vol. 209, pp. 3953-3963, 2006.

[18] D. A. Neumann, Kinesiology of the Musculoskeletal System: Foundation for Physical Rehabilitation. St. Louis, MO, USA: Mosby, 2002.

[19] F. E. Zajac, R. R. Neptune, and S. A. Kautz, "Biomechanics and muscle coordination of human walking - Part II: Lessons from dynamical simulations and clinical implications," Gait Posture, vol. 17, pp. 1-17, 2003.

[20] A. D. Kuo, "The six determinants of gait and the inverted pendulum analogy: A dynamic walking perspective," Hum. Mov. Sci., vol. 26, pp. 617656, 2007.

[21] D. A. Kuo, J. M. Donelan, and A. Ruina, "Energetic consequences of walking like an inverted pendulum: Step-to-step transitions," Exerc. Sport Sci. Rev., vol. 3, pp. 88-97, 2005.

[22] H. Herr and M. Popovic, "Angular momentum in human walking," J. Exp. Biol., vol. 211, pp. 467-481, 2008.

[23] B. Mariani, C. Hoskovec, S. Rochat, C. Büla, J. Penders, and K. Aminian, "3-D gait assessment in young and elderly subjects using foot-worn inertial sensors," J. Biomech., vol. 43, no. 15, pp. 2999-3006, 2010.

[24] G. A. Cavagna, H. Thys, and A. Zamboni, "The sources of external work in level walking and running," J. Physiol., Lond., vol. 262, pp. 639-657, 1976

[25] C. Y. Lee and J. J. Lee, "Estimation of walking behavior using accelerometers in gait rehabilitation," Int. J. Human-friendly Welf. Robot. Syst., vol. 3, no. 2, pp. 32-36, 2002.

[26] H. Vathsangam, A. Emken, D. Spruijt-Metz, and G. S. Sukhatme, "Toward free-living walking speed estimation using Gaussian process based regression with on-body accelerometers and gyroscopes," in Proc. 4th Int. Conf. NO PERMISSIONS Pervasive Comput. Technol. Healthcare (PervasiveHealth), Mar. 2010, pp. 1-8.

[27] H. Vathsangam, A. Emken, D. Spruijt-Metz, and G. S. Sukhatme, "The unscented Kalman filter," in Kalman Filtering and Neural Networks. New York, NY, USA: Wiley, 2001, ch. 7.
[28] S. Julier and J. Uhlmann, "A new extension of the Kalman filter to nonlinear systems," Proc. SPIE, vol. 3068, pp. 182-193, 1997.

[29] Q. Li, M Young, V Naing, and J. M. Donelan, "Walking speed and slope estimation using shank-mounted inertial measurement units," J. Biomech., vol. 43, no. 8, pp. 1640-1643, May 2010

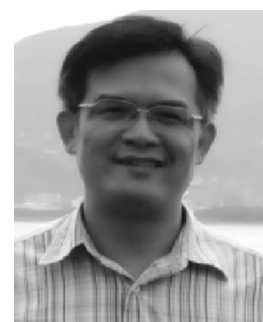

Jwu-Sheng Hu (M'94) received the B.S. degree from the Department of Mechanical Engineering, National Taiwan University, Taipei City, Taiwan, in 1984, and the M.S. and Ph.D. degrees from the Department of Mechanical Engineering, University of California at Berkeley, Berkeley, CA, USA, in 1988 and 1990, respectively.

From 1991-1993, he was an Assistant Professor in the Department of Mechanical Engineering, Wayne State University, Detroit, MI, USA, In 1993 , he joined the Department of Electrical Engineering, National Chiao Tung University, Hsinchu, Taiwan, and became a Full Professor in 1998 and has been serving as the Vice-Chairman of the Department since 2006. Since 2008, he has been working in-part at the Industrial Technology Research Institute (ITRI), Hsinchu, where he serves as the Advisor for the Intelligent Robotics program and Principle Investigator of several join research projects on robotics across academia, industry, and research institutions. He also serves as a Research Faculty at the National Chip Implementation Center (CIC) of Taiwan for embedded system design applications. His current research interests include robotics, microphone array, mechatronics, and embedded systems.

He received the Research Initiation Award from the National Science Foundation. He is now the Chairman of the Taipei Chapter, IEEE RA Society.

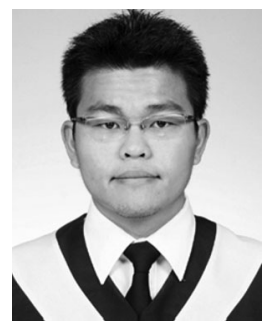

Kuan-Chun Sun was born in Kaohsiung, Taiwan, in 1985 . He received the B.S. degree and the M.S. degree in electrical and control engineering from $\mathrm{Na}$ tional Chiao Tung University, Taiwan, in 2008 and 2009 respectively, where he is currently working toward the Ph.D. degree in the Department of Electrical and Control Engineering, National Chiao Tung University, Hsinchu, Taiwan.

His research interests include signal processing, IMU sensor fusion, robotics, Kalman filtering, $\mathrm{H}$ infinity filtering, optimization.

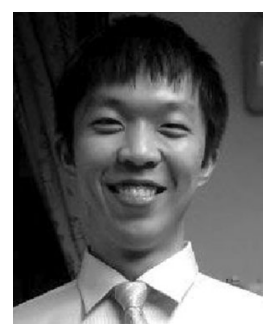

Chi-Yuan Cheng was born in Taipei, Taiwan, in 1989. He received the B.S. degree in electrical and control engineering from National Chiao Tung University, Hsinchu, Taiwan, in 2011, where he is currently working toward the M.S. degree from the Department of Electrical and Control Engineering.

His research interests include human gait estimation, GPS/IMU integrated navigation system, and adaptive noise estimation. 